\title{
Características clínico-moleculares del cáncer gástrico cardial asociado al virus Epstein Barr
}

\author{
Alejandro Corvalán $R^{1,2}$, Suminori Akiba ${ }^{3}$, \\ María Teresa Valenzuela $B^{4}$, Miguel Angel Cumsille $G^{4, a}$, \\ Chihaya Koriyama ${ }^{3}$, Jorge Argandoña $C^{2, b}$, \\ Claudia Backhouse $\mathrm{E}^{2, \mathrm{C}}$, Matilde Bal $\mathrm{C}^{4}$, \\ Fernando Mena U ${ }^{5}$, Mariana Palma V ${ }^{2, c}$, Yoshito Eizuru ${ }^{3}$. \\ Clinical and molecular features of cardial
gastric cancer associated to Epstein Barr virus
}

Background: Mortality caused by cardial gastric cancer in Chile, is increasing. Previously we demonstrated an association between Epstein Barr virus and this specific location of gastric cancer. Aim: To perform a clinical and molecular characterization of cardial gastric cancer associated to Epstein Barr virus. Material and methods: Epstein Barr virus was identified in 93 cardial gastric tumors, by in situ hybridization. Clinical and pathological features, survival and expression of p53 and c-erbB2 were compared between tumors with or without the presence of the virus. Results: Twenty two (23.6\%) tumors expressed Epstein Barr virus. No difference in sex or age of patients with tumors positive or negative for the virus was observed. Epstein Barr positive tumors had a tendency to have a higher frequency of Bormann III endoscopic appearance and a lower frequency of $\mathrm{p} 53$ accumulation $(\mathrm{p}=0.06)$. Five years survival was $67 \%$ and $42 \%$ of tumors positive and negative for the presence of the virus, respectively $(p=0.57)$. Conclusions: Our results, although not significant, show a tendency towards unique characteristics of cardial gastric tumors associated to Epstein Barr virus (Rev Méd Chile 2005; 133: 753-60).

(Key Words: Epstein-Barr virus infections; Herpes virus 4, human; Stomach neoplasms)

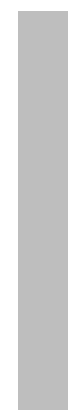
Recibido el 1 de septiembre, 2004. Aceptado en versión corregida el 30 de marzo, 2005.
Trabajo financiado por Grants in Aid for Scientific Research (12218231) e International Scientific Research, Special Cancer Research (09042007) Ministerio de Educación, Japón y Dirección Académica, Clínica Las Condes, Chile (Proyecto Her2/neu y Cáncer Gástrico de ubicación cardial).
${ }^{1}$ Departamento de Anatomía Patológica, Pontificia Universidad Católica de Chile. ${ }^{2}$ Instituto Chileno Japo- nés de Enfermedades Digestivas, Hospital Clínico San Borja Amiarán, Santiago, Chile. ${ }^{3}$ Departamento de Salud Pública y Centro de Enfermedades Virales Crónicas, Facultad de Medicina, Universidad de Kagoshima, Kagoshima, Japón. ${ }^{4}$ Escuela de Salud Pública, Facultad de Medicina, Universidad de Chile, Santiago Chile. ${ }^{5}$ Departamento de Patología, Hospital Max Peralta, Cartago, Costa Rica.
aMagíster en Bioestadística
bLicenciado en Tecnología Médica
'Tecnóloga Médica

Correspondencia a: Dr. Alejandro Corvalán R. Laboratorio Biología Molecular, Departamento de Anatomía Patológica, Facultad de Medicina, Pontificia Universidad Católica de Chile. Lira 85, Santiago. Casilla 114-D. Teléfono: 56(2) 3543209. Fax: 56(2) 6395101. E-mail: corvalan@med.puc.cl 
$\mathrm{E}^{1}$ cáncer gástrico (CG) representa la primera causa de muerte por enfermedades neoplásicas en Chile y aunque su tasa de mortalidad se ha estabilizado ${ }^{1}$, la frecuencia del CG de ubicación cardial se encuentra en aumento ${ }^{2-4}$. En efecto, autores chilenos ${ }^{2,3}$ han demostrado que los tumores cardiales han incrementado entre 12 y 15\% en los últimos 30 años, llegando a representar el $42,3 \%$ de las localizaciones. De modo similar, investigadores americanos ${ }^{4}$ han reportado un incremento anual de 4,4\% entre 1976 y 1987, sin observar modificaciones en la frecuencia del CG, en otras localizaciones. Se ha postulado que el CG cardial, sería una forma particular de CG, dado que se comunica una peor sobrevida a 5 años en esta localización en comparación con CG de otras ubicaciones ${ }^{5}$. Por otra parte, se ha descrito que la frecuencia de mutaciones de los oncogenes p53 y Her2/neu sería diferente entre CG cardial y antral ${ }^{6,7}$ y Hansen et $\mathrm{al}^{8}$ han señalado que Helicobacter pylori no tendría un rol tan relevante en la patogénesis del CG cardial como el observado en la ubicación antral. Incluso se ha observado un probable rol protector de Helicobacter pylori en el desarrollo del CG cardial ${ }^{9}$. Tomadas en conjunto, estas observaciones sugieren que el CG cardial sería una forma emergente y particular de CG.

El virus de Epstein-Barr (VEB), tradicionalmente asociado a neoplasias linfoides ${ }^{10}$, también ha sido descrito en tumores epiteliales como carcinoma nasofaríngeo y linfoepiteliomas de distintos órganos, incluyendo el estómago ${ }^{11,12}$. En esta ubicación, los linfoepiteliomas no representan más de $5 \%$ de los tumores gástricos ${ }^{13}$, sin embargo, en los últimos años se ha demostrado una emergente asociación entre VEB y CG ${ }^{14}$. En efecto, diversas publicaciones señalan una asociación de $6,9 \%$ a $18 \%$ entre VEB y CG $^{15-17}$ con características clínico-patológicas únicas, como predominio en hombres, localización alta (cardias y tercio medio del estómago) y una frecuencia similar de los subtipos "intestinal" y "difuso"18. Recientemente hemos analizado las características clínico-patológicas del CG asociado a VEB en Chile, encontrando una asociación de 16,8\% (31/185), una de las más altas del mundo, y un perfil clínico-patológico único ${ }^{19}$.
Este perfil se caracteriza por una distribución similar entre ambos sexos, una fuerte asociación con ubicación cardial y un predominio del patrón histológico "difuso"19. Dado que estas características han sido descritas en México ${ }^{20} \mathrm{y}$ en descendientes mexicanos en Estados Uni$\operatorname{dos}^{21}$, hemos sugerido la presencia de un perfil único del CG asociado a VEB en Latinoamérica ${ }^{19}$.

Ya que el CG cardial representa una entidad propia y emergente en Chile y que una de las principales características del CG asociado a VEB es la fuerte localización cardial, el objetivo de nuestro estudio fue analizar características clínicopatológicas, moleculares y de sobrevida del CG cardial en sus formas asociada y no asociada al VEB.

\section{MATERIALES Y MÉTODOS}

Pacientes. El estudio se realizó sobre la base de 305 piezas quirúrgicas de pacientes operados por CG en el Servicio de Cirugía del Hospital San Borja Arriarán y archivados en el Instituto Chileno-Japonés de Enfermedades Digestivas del mismo hospital entre 1993 y 1999. De este material y utilizando la definición de Locke et $\mathrm{al}^{22}$ que definen como mucosa gástrica cardial a la región entre la unión gastroesofágica y los primeros 5 cm de estómago, se identificaron 93 casos, que son la base del presente estudio. Las características clínicas de estos casos se obtuvieron de la revisión de fichas médicas y las características patológicas de informes anátomo-patológicos correspondientes. Se consignó edad, sexo, tamaño tumoral $(<5$ y $>5 \mathrm{~cm})$, infiltración de pared gástrica y compromiso ganglionar (sin consignar número de ganglios comprometidos) de acuerdo a la Unión Internacional contra el Cáncer ${ }^{23}$ y tipo histológico de acuerdo a la clasificación de Lauren ${ }^{24}$. En los tumores que infiltraban muscular propia o serosa, se consignó además la forma macroscópica según la clasificación de Bormann ${ }^{25}$.

Hibridación in situ para la identificación del virus de Epstein-Barr. La presencia de VEB se realizó determinando la expresión del ARN pequeño no poliadenilado intranuclear-1 (EBER-1), 
el producto viral más abundante de la infección latente de $\mathrm{VEB}^{26}$. La determinación se realizó con la sonda TTGCTAGGGAGGAGACGTGT complementaria a los nucleótidos 6653-6672 del gen EBER-1 de acuerdo al protocolo descrito por Chang et $\mathrm{al}^{27}$.

Inmunohistoquímica para la identificación de p53 y Her2/neu. La determinación de p53 se realizó por inmunohistoquímica utilizando el anticuerpo DO-7 (Dako) de acuerdo a protocolos establecidos $^{28}$. Brevemente, se realizaron cortes de $4 \mu \mathrm{m}$ que fueron montados en portaobjetos seguidos de desparafinación, rehidratación y digestión con Tripsina al 0,37\%. A continuación, las muestras fueron incubadas con el anticuerpo DO-7 (1:10) a $4^{\circ} \mathrm{C}$ por $12 \mathrm{~h}$ seguidas de anticuerpo secundario conjugado con biotina (1:300) por $30 \mathrm{~min}$ a $37^{\circ} \mathrm{C}$ y complejo estreptavidina-peroxidasa (1:300) por $30 \mathrm{~min}$ a $37^{\circ} \mathrm{C}$. Finalmente, las láminas fueron incubadas con diaminobenzidina (DAB) por $10 \mathrm{~min}$ a temperatura ambiente en presencia de $\mathrm{H}_{2} \mathrm{O}_{2}$ al $0,3 \%$ y contrateñidas con hematoxilina. La determinación de Her-2/neu se realizó con el anticuerpo policlonal pAB-1 (Dako) en condiciones similares a las descritas y considerando reacción positiva a la tinción específica en la membrana citoplasmática de células tumorales ${ }^{29}$.

Estadística. Los resultados y las asociaciones con variables clínico-patológicas se estudiaron usando el método de chi-cuadrado $\left(\mathrm{x}^{2}\right)$ y los análisis de sobrevida usando el método de Kaplan-Meier. Estos análisis estadísticos se hicieron en el paquete computacional Stata 6.0.

\section{Resultados}

Hibridación in situ. La hibridación in situ se realizó en 93 casos, resultando $22(23,6 \%)$ con tinción positiva. En los casos considerados con tinción positiva se observó la expresión de EBER-1 en forma uniforme y exclusiva en todos los núcleos de las células tumorales y no se observó expresión de EBER-1 en células epiteliales no tumorales ni linfocitos peritumorales (Figura 1).
Características clínicas y patológicas. Los resultados de la comparación de variables clínicas y anátomo-patológicas entre casos de CG cardial VEB + y VEB- se muestran en la Tabla 1. Se observa que con relación al sexo y edad no hay diferencias significativas entre ambos grupos. Sin embargo, la edad más frecuente en que ocurrió la enfermedad, o modo, fue menor en el subtipo VEB+ (52 años vs 67 años). Con relación a variables anátomo-patológicas se observa que no hay diferencias en el tamaño tumoral como tampoco la infiltración de la pared gástrica entre los tumores VEB+ y VEB-. Entre los tumores T2-T4 (avanzados) observamos que aunque la forma Bormann III fue la más frecuente para ambos grupos, esta presentación macroscópica fue más frecuente en los tumores EBV+ $(p=0,06)$. Con relación a la presentación histológica y basándose en la clasificación de Lauren, no se observaron diferencias entre los tipos "intestinal" y "difuso". En esta serie observamos 4 casos con abundante infiltrado linfocitario, los que fueron clasificados como linfoepitelioma gástrico y todos ellos correspondieron al grupo VEB+.

Características moleculares. La determinación de p53 se realizó en 21 casos VEB+ y en 57 casos VEB-. Se consideró tinción positiva con $10 \%$ o más

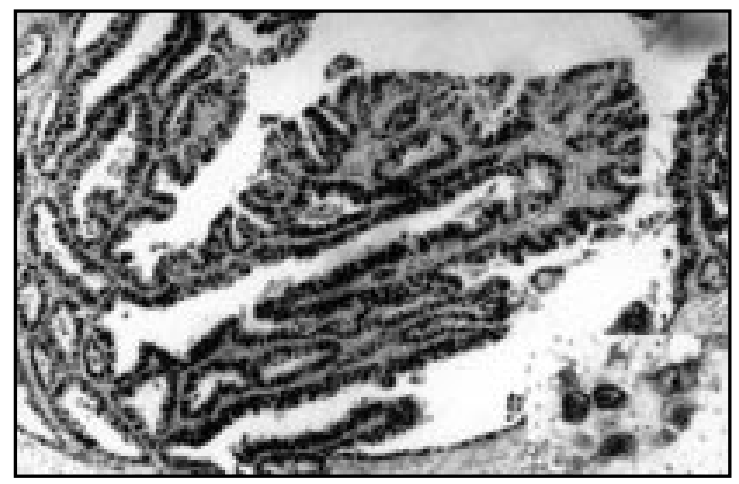

FIgURA 1. Hibridación in situ para EBER-1. Se observa expresión uniforme y exclusiva de EBER-1 en todos los núcleos de células tumorales de un cáncer gástrico cardial de tipo intestinal bien diferenciado (papilo-tubular), indicando la presencia de VEB (40X). Inserto: detalle con mayor aumento de la tinción nuclear. 


\section{Tabla 1. C orrelaciones clínico-patológicas del cáncer gástrico de ubicación cardial asociado al virus de Epstein-Barr}

\begin{tabular}{|c|c|c|c|c|c|}
\hline & & $\begin{array}{l}\text { itivo } \\
\text { os }\end{array}$ & $\begin{array}{r}\text { VEF } \\
7\end{array}$ & $\begin{array}{l}\text { ativo } \\
\text { S }\end{array}$ & \\
\hline & $\mathrm{n}$ & $(\%)$ & $\mathrm{n}$ & (\%) & $\mathrm{p}$ \\
\hline Sexo & & & & & \\
\hline hombre & 6 & $(27,3)$ & 25 & $(35,2)$ & \\
\hline mujer & 16 & $(72,7)$ & 46 & $(64,8)$ & 0,49 \\
\hline Edad & & & & & \\
\hline promedio & $63,2 \mathrm{a}$ & (26-79a) & $61,8 \mathrm{a}$ & (20-79a) & \\
\hline Tamaño tumor & & & & & \\
\hline$<5 \mathrm{~cm}$ & 4 & $(22,2)$ & 26 & $(42,6)$ & \\
\hline$>5 \mathrm{~cm}$ & 14 & $(77,8)$ & 35 & $(57,4)$ & 0,12 \\
\hline Pared gástrica & & & & & \\
\hline T0-T1 & 3 & $(15,8)$ & 9 & $(12,9)$ & \\
\hline $\mathrm{T} 2-\mathrm{T} 4$ & 16 & $(84,2)$ & 61 & $(87,1)$ & 0,71 \\
\hline Bormann I & 0 & & 4 & $(6,6)$ & \\
\hline Bormann II & 2 & $(12,5)$ & 19 & $(31,1)$ & \\
\hline Bormann III & 11 & $(68,8)$ & 26 & $(42,6)$ & $0,06^{\mathrm{a}}$ \\
\hline Bormann IV & 2 & $(12,5)$ & 9 & $(14,8)$ & \\
\hline Bormann V & 1 & $(6,2)$ & 3 & $(4,9)$ & \\
\hline Linfonodos & & & & & \\
\hline negativo & 8 & $(42,1)$ & 19 & $(37,3)$ & \\
\hline positivo & 11 & $(57,9)$ & 32 & $(62,7)$ & 0,71 \\
\hline Histología & & & & & \\
\hline intestinal & 13 & $(59,1)$ & 51 & $(71,8)$ & \\
\hline difuso & 9 & $(40,9)$ & 20 & $(28,2)$ & 0,26 \\
\hline
\end{tabular}

aBormann III vs Bormann I, II, IV y V.

de células tumorales positivas a nivel nuclear (Figura 2) y los resultados se muestran en la Tabla 2. Se observa que sólo $3(14,2 \%)$ de 21 tumores VEB+ presentaron acumulación de p53. Por el contrario, entre los tumores VEB-, $21(36,8 \%)$ de 57 casos VEB- acumularon p53 $(\mathrm{p}=0,06)$. La determinación de Her2/neu se realizó en 20 casos VEB+ y 61 casos VEB-. Se consideró tinción positiva la tinción de membrana citoplasmática exclusivamente en las células tumorales (Figura 3). Los resultados se muestran en la Tabla 2. No observamos expresión de Her2/neu en ningún caso de CG cardial VEB+, y sólo en $3(4,9 \%)$ casos VEB-.

Análisis de sobrevida. De 93 pacientes portadores de CG cardial, los datos de sobrevida fueron

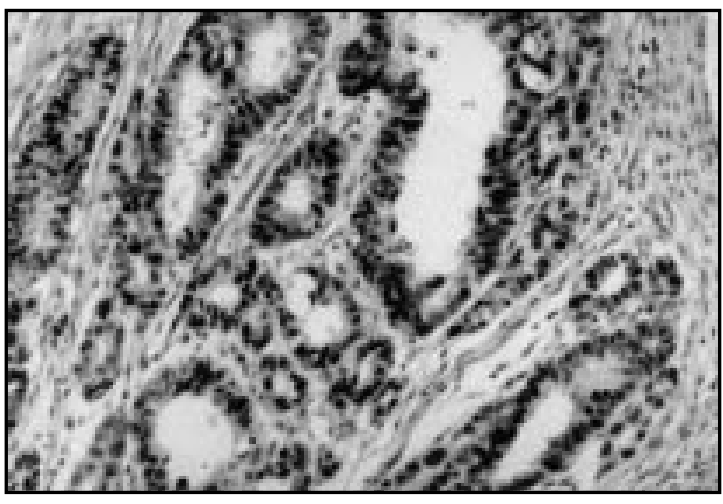

FiguRA 2. Inmunohistoquímica para proteína tumoral p53. Se observa tinción de p53 en el núcleo de células tumorales (40X). 
Tabla 2. Correlaciones moleculares del cáncer gástrico cardial asociado al virus de Epstein-Barr

\begin{tabular}{|crrcc|}
\hline & $\begin{array}{c}\text { VEB positivo } \\
\mathrm{n}\end{array}$ & $\begin{array}{c}\text { VEB negativo } \\
\mathrm{n}(\%)\end{array}$ & $\mathrm{p}$ \\
\hline $\begin{array}{c}\text { p53 } \\
\text { negativo }\end{array}$ & 18 & $(85,7)$ & $36(63,2)$ & \\
positivo & 3 & $(14,3)$ & $21(36,8)$ & 0,06 \\
$\begin{array}{c}\text { Her2/neu } \\
\text { negativo } \\
\text { positivo }\end{array}$ & 20 & $(100)$ & $58(95,1)$ & \\
& 0 & 0 & $3(4,9)$ & 0,17 \\
\hline
\end{tabular}

obtenidos en 77 casos, 18 VEB+ y 59 VEB-. Observamos una sobrevida global de $67 \%$ y $42 \%$ para los tumores VEB+ y VEB-, respectivamente. La tendencia en el riesgo relativo de morir fue $0,57(95 \%$ IC $=0,23$ a 1,$41 ; \mathrm{p}=0,23)$ para los tumores VEB- con respecto a los tumores VEB+. Para comparar la probabilidad de sobrevida en tiempos específicos entre tumores EBV+ y EBV-, realizamos un análisis de función de sobrevida (Tabla 3), que nos muestra que los tumores EBV+ tienen una mejor sobrevida inicial, pero que estas diferencias tienden a desaparecer después de los 48 meses de seguimiento.

\section{DisCUSIÓN}

Varias líneas de evidencias apoyan la asociación entre VEB y CG. La demostración de monoclonalidad del genoma de VEB en casos de CG indica que la infección viral precede a la expansión neoplásica ${ }^{30}$. Por otra parte, la presencia de anticuerpos anti-VEB elevados en pacientes con CG asociado a VEB respecto a pacientes con CGVEB negativo o sujetos controles ${ }^{30-32}$, y la presencia del patrón histológico denominado lace pattern ${ }^{33}$, característico de esta forma tumoral, son otras evidencias que apoyan esta asociación.

Previamente hemos identificado una fuerte relación entre VEB y CG de ubicación cardial ${ }^{19}$. Para caracterizar esta asociación, analizamos características clínico-patológicas, moleculares y de sobrevida en una serie de 93 casos de CG de

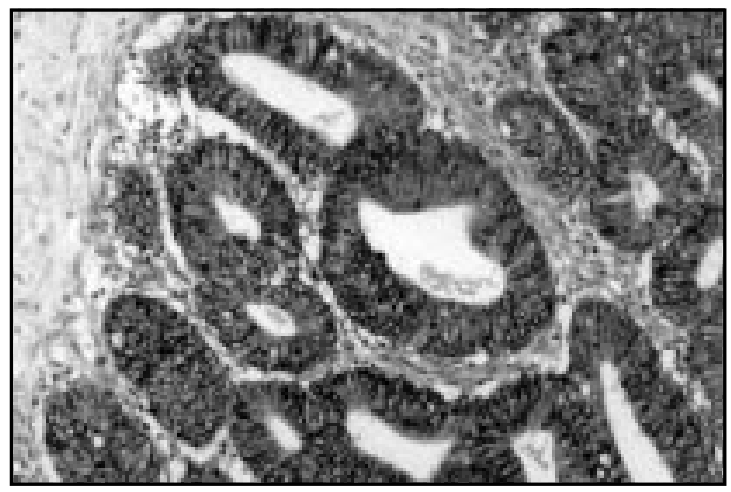

FIgURA 3. Inmunohistoquímica para oncogen c-erbB2. Se observa tinción en la membrana citoplasmática de células tumorales (40X).

Tabla 3. Análisis de la función de sobrevida para el virus Epstein-Barr en cáncer gástrico cardial

\begin{tabular}{|lcc|}
\hline Tiempo & VEB- & VEB+ \\
\hline 1 mes & $100 \%$ & $100 \%$ \\
12 meses & $78,1 \%$ & $94,4 \%$ \\
24 meses & $63,7 \%$ & $88,8 \%$ \\
36 meses & $57,1 \%$ & $76,1 \%$ \\
48 meses & $52,4 \%$ & $69,8 \%$ \\
60 meses & $52,4 \%$ & $62,8 \%$ \\
72 meses & $52,4 \%$ & $62,8 \%$ \\
\hline
\end{tabular}

ubicación cardial de los cuales $22(22,6 \%)$ correspondieron al tipo asociado a VEB. Al comparar las características anátomo-patológicas, llama la atención la mayor frecuencia de presentación Bormann III en los tumores VEB+. Esta observación no ha sido descrita previamente, sólo Yanai et $\mathrm{al}^{34}$ han señalado que la presentación endoscópica más frecuente del CG asociado a VEB sería la incipiente IIc. Por otra parte, ambos tipos histológicos de Lauren estuvieron representados en proporciones similares. Esta observación es contraria a la asociación descrita con el tipo "difuso"19 y probablemente indica que la localización y el tipo histológico serían variables independientes en el CG asociado a VEB.

La proteína p53 es una de las más importantes en carcinogénesis gástrica ${ }^{35}$. Esta proteína es de ubicación nuclear, es activada en respuesta a daño celular y su inactivación se asocia al 
desarrollo de neoplasias ${ }^{36}$. La inactivación, por mutaciones puntuales o complejos con proteínas virales estabiliza a p53, con aumento de su vida media, característica que permite el uso de la inmunohistoquímica para su detección ${ }^{37}$. Utilizando esta metodología, la frecuencia de acumulación de p53 varía entre $23 \%$ y $61 \% 37$. Nuestros resultados muestran una tendencia a una baja frecuencia de acumulación de p53 en tumores asociados a VEB, los cuales son concordantes con la literatura ${ }^{38,39}$ aunque en estos trabajos no se hace referencia a la ubicación cardial. C-erbB2 es un oncogen que codifica para receptores de factores de crecimiento y está activado por amplificación génica ${ }^{29}$. La amplificación de c-erbB2 es considerado el factor pronóstico molecular más importante en CG, ya que se correlaciona con invasión serosa y linfática, metástasis hepática y peritoneal y menor sobrevida a 5 años ${ }^{40}$. Sin embargo, no hay estudios que analicen el rol de c-erbB2 en CG asociado a VEB y nuestros resultados no demuestran una relación entre ambos, al menos en ubicación cardial.

\section{REFERENCIAS}

1. Serra I, Báez S, Serra J, Calvo A, Decinti e. Evolución epidemiológica reciente del cáncer gástrico en Chile y el mundo. Rev Chil Cir 1997; 49: 54-63.

2. Csendes A, Smok G, Medina E, Salgado I, Rivera R, Quitral M. Características evolutivas del cáncer gástrico 1958-1990. Rev Méd Chile 1992; 120: 3642.

3. Duarte I, Ohmke J, Ciani S, Vilarroel L Patrones de carcinoma en gastrectomías de adultos chilenos: Estudio multivariado en un país de alto riesgo. Gastr Latinoam 2001; 12: 12-8.

4. Blot WJ, Devesa SS, Kneler RW, Fraumeni JF Jr. Rising incidence of adenocarcinoma of the esophagus and gastric cardia. JAMA 1991; 265: 1287-9.

5. Ohno S, Tomisaki S, Oiwa H, Sakaguchi $Y$, Ichiyoshi $Y$, MaEHARA Y ET AL. Clinicopathologic characteristics and outcome of adenocarcinoma of the human gastric cardia in comparison with carcino-
Con relación a sobrevida, aunque no observamos diferencias estadísticamente significativas, en la sobrevida entre los tumores VEB+ y VEB-, sí observamos una tendencia a mejor pronóstico en los tumores EBV+. Esta información es concordante con la descrita por van Beek et $\mathrm{al}^{41}$, en población caucásica y por nuestro propio grupo en una serie de 192 CG japoneses ${ }^{42}$. Dado que la ubicación cardial se considera de peor pronósti$\mathrm{CO}^{5}$, la observación de una tendencia a mejor pronóstico en los tumores EBV+, sería sugerente de un potencial rol específico de EBV+ en la historia natural del cáncer gástrico cardial. El análisis de la función de sobrevida, indica que la presencia de EBV sería particularmente relevante en los primeros 48 meses de seguimiento de estos pacientes.

En resumen, nuestros resultados, aunque no significativos, muestran tendencias de asociaciones clínico-moleculares y de sobrevida del CG cardial asociado a VEB. Estos resultados aportan información adicional a la caracterización del rol de VEB en CG cardial, un forma emergente de CG.

ma of other regions of the stomach. J Am Coll Surg 1995; 180: 577-82.

6. Flejou JF, Gratio V, Muzeau F, Hamelin R. p53 abnormalities in adenocarcinoma of the gastric cardia and antrum. Mol Pathol 1999; 52: 263-8.

7. Albino AP, Jaehne J, Altorki N, Blundell M, URMaCHER C, LAUWERS G ET aL. Amplification of HER-2/neu gene in human gastric adenocancinomas: correlation with primary site. Eur J Surg Oncol 1995; 21: 56-60.

8. Hansen S, Melby KK, Aase S, Jelum E, Voldset SE. Helicobacter pylori infection and risk of cardia cancer and non-cardia gastric cancer. A nested case-control study. Scand J Gastroenterol 1999; 34: 353-60.

9. Chow WH, Blaser MJ, Blot WJ, Gammon MD, Vaughan TL, Rusch HA ET aL. An inverse relation between cagA+ strains of Helicobacter pylori infection and risk of esophageal and gastric cardia adenocarcinoma. Cancer Res 1998; 58: 588-90. 
10. Rickinson A, KiefF E. Epstein-Barr virus. In: Fields B, Knipe D, Howley P, eds. Fields Virology. $3^{\underline{a}}$ ed. Philadelphia: Lippincont-Raven Publishers, 1996; 2397-445.

11. Oda K, Tamaru J, Takenouchi T, Mikata A, NunomuRA M, SAIToH N et aL. Association of Epstein-Barr virus with gastric carcinoma with lymphoid stroma. Am J Pathol 1993; 143: 1063-71.

12. HAUSEN HZ. Epstein-Barr virus in human tumor cells. Int Rev Exp Pathol 1972; 11: 233-58.

13. Watanabe $H$, Enjoji M, Imai T. Gastric carcinoma with lymphoid stroma. Its morphologic characteristics and prognostic correlations. Cancer 1976; 38: 232-43.

14. Shibata D, Weiss LM. Epstein-Barr virus associated gastric adenocarcinoma. Am J Pathol 1992; 140: 769-74.

15. Tokunaga M, Land CE, Uemura Y, Tokudome T, TanaKa S, Sato E. Epstein-Barr virus in gastric carcinoma. Am J Pathol 1993; 143: 1250-4.

16. Galetsky SA, Tsvetnov VV, Land CE, Afanasieva TA, Petrovichev NN, Gurtsevitch VE et al. Epstein-Barr virus associated gastric cancer in Russia. Int $\mathrm{J}$ Cancer 1997; 73: 786-9.

17. Ott G, Kirchner T, MuLer-Hermelink HK. Monoclonal Epstein-Barr virus genomes but lack of EBV-related protein expression in different types of gastric carcinoma. Histopathology 1994; 25: 323-9.

18. TAKADA K. Epstein-Barr virus and gastric carcinoma. Mol Pathol 2000; 53: 255-61.

19. Corvalán A, Koriyama C, Akiba S, Eizuru Y, Backhouse C, Palma M et al. Epstein-Barr virus in gastric carcinoma is associated with location in the cardia and with a diffuse histology: a study in one area of Chile. Int J Cancer 2001; 94: 527-30.

20. Herrera-Goepfert R, Reyes E, Hernández-Avila M, Mohar A, Shinkura R, Fujiyama C et al. Epstein-Barr virus-associated gastric carcinoma in Mexico: analysis of 135 consecutive gastrectomies in two hospitals. Mod Pathol 1999; 12: 873-8.

21. Guliey ML, Pulitzer DR, Eagan PA, Schneider BG. Epstein-Barr virus infection is an early event in gastric carcinogenesis and is independent of bcl2 expression and p53 accumulation. Hum Pathol 1996; 27: 20-7.

22. Locke G, TaLiey $N$, Carpenter $H$, Harmsen $W$, Zinsmeister A, Melton L Changes in the site and histology specific incidence of gastric cancer during a 50 years period. Gastroenterology 1995; 109: 1750-6.

23. Fenoglio-Preiser C, Carneiro F, Correa P, Guilford P, Lambert B, Megraud F et al. Gastric carcinoma. In: Hamilton HB, Aaltonen L, eds. Pathology and Genetics of Tumours of the Digestive System. Lyon: IARC Press, 2000.

24. LAUREN P. The two histological main types of gastric carcinoma, diffuse and so-called intestinal -type carcinoma. Acta Path Microbiol Scan 1965; 64: 31-49.

25. Bormann R. Geschwulse des magens und duodenums. In: Henske F, Lubarsch O, eds. Handbuch der speziellen pathologischen antomie und histologie. Volume IV-L Berlin: Julis Springler, 1926; 864-71.

26. Takada K, Nanbo A. The role of EBERs in oncogenesis. Semin Cancer Biol 2001; 11: 461-7.

27. Chang KL, Chen YY, Shibata D, Weiss LM. Description of an in situ hybridization methodology for detection of Epstein-Barr virus RNA in paraffinembedded tissues, with a survey of normal and neoplastic tissues. Diagn Mol Pathol 1992; 1: 24655.

28. Kaserer K, Schmaus J, Bethge U, Migschitz B, FAsching S, WaLCh A ET AL. Staining patterns of p53 immunohistochemistry and their biological significance in colorectal cancer. J Pathol 2000; 190: 450-6.

29. Ross JS, McKenna BJ. The HER-2/neu oncogene in tumors of the gastrointestinal tract. Cancer Invest 2001; 19: 554-68.

30. Imai S, Koizumi S, Sugiura M, Tokunaga M, Uemura Y, Y Амамото N Eт AL. Gastric carcinoma: monoclonal epithelial malignant cells expressing EpsteinBarr virus latent infection protein. Proc Natl Acad Sci USA 1994; 91: 9131-5.

31. Levine PH, Stemmermann G, Lennette ET, Hildesheim A, Shibata D, Nomura A. Elevated antibody titers to Epstein-Barr virus prior to the diagnosis of Epstein-Barr virus associated gastric adenocarcinoma. Int J Cancer 1995; 60: 642-4.

32. Shinkura R, Yamamoto N, Koriyama C, Shinmura $Y$, Eizuru Y, Tokunaga M. Epstein-Barr virus specific antibodies in Epstein-Barr virus positive and negative gastric carcinoma cases in Japan. J Med Virol 2000; 60: 411-6.

33. Uemura $\mathrm{Y}$, Tokunaga M, Arikawa J, Yamamoto N, Hamasaki Y, TANaKa S ET al. A unique morphology 
of Epstein-Barr virus related early gastric carcinoma. Cancer Epidemiol Biomarkers Prev 1994; 3: 607-11.

34. Yanai H, Nishikawa J, Mizugaki $Y$, Shimizu N, Takada K, Matsusaki K et al. Endoscopic and pathologic features of Epstein-Barr virus associated gastric carcinoma. Gastrointest Endosc 1997; 45: 236-42.

35. Convalán A. Genética molecular del cáncer gástrico. In: Csendes A, ed. Actualizaciones en cáncer gástrico. Santiago: Editorial Mediterráneo (en prensa).

36. Hainaut P, HoLSTein M. p53 and human cancer: the first ten thousand mutations. Adv Cancer Res 2000; 77: 81-137.

37. Gabbert H, MuLer W, Schneider A, Meier S, Hommel G. The relationship of p53 expression to the prognosis of 418 patients with gastric carcinoma. Cancer 1995; 76: 720-6.

38. Ojima $H$, Fukuda T, Nakajima T, Nagamachi $Y$. Infrequent overexpression of p53 protein in
Epstein-Barr virus-associated gastric carcinomas. Jpn J Cancer Res 1997; 88: 262-6.

39. Leung SY, Chau KY, Yuen ST, Chu KM, Branicki FJ, CHung LP. p53 overexpression is different in Epstein-Barr virus associated and Epstein-Barr virus negative carcinoma. Histopathology 1998; 33: 311-7.

40. Yonemura Y, Ninomiya I, Yamaguchi A, Fushida S, Kimura H, Ohoyama $S$ et al. Evaluation of immunoreactivity for erbB-2 protein as a marker of poor short term prognosis in gastric cancer. Cancer Res 1991; 51: 1034-8.

41. van Beek J, zur Hausen A, Kiein Kranenbarg E, van de Velde CJH, Middeldorp JM, van den Brule AJC et AL. EBV-Positive Gastric Adenocarcinomas: A Distinct Clinicopathologic Entity With a Low Frequency of Lymph Node Involvement. J Clin Oncol 2004; 22: 664-70.

42. Koriyama C, Aкiba S, Itoh T, Kijima Y, Sueyoshi K, Convalán A et al. Prognostic significance of Epstein-Barr virus involvement in gastric carcinoma in Japan. Int J Mol Med 2002; 10: 635-9. 